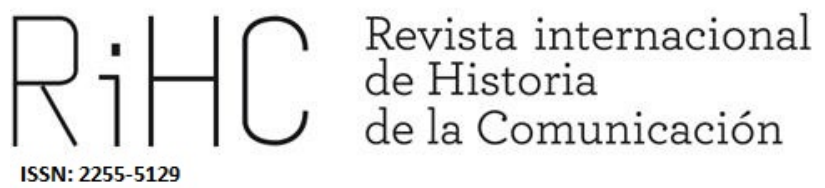

\title{
ANÁLISIS DE LOS PRIMEROS ESTUDIOS SOBRE EL LIDERAZGO PERSONAL Y LA DIFUSIÓN DE TENDENCIAS DE MODA (1950-2000)
}

\section{Analysis of the first studies on Personal Leadership and Fashion Innovation Difussion (1950-2000)}

DOI: http://dx.doi.org/10.12795/RiHC.2018.i10.06

Recibido: $28 / 03 / 2018$

Aceptado: $24 / 05 / 2018$

Publicado: $25 / 06 / 2018$

Patricia SanMiguel (iD https://orcid.org/0000-0002-0438-2830

ISEM Fashion Business School-Universidad Navarra, patricia.sanmiguel@isem.es

Teresa Sádaba (iD https://orcid.org/0000-0002-2441-5724

ISEM Fashion Business School-Universidad Navarra, teresa.sadaba@isem.es

Resumen: Los estudios sobre el liderazgo de opinión comenzaron con las aportaciones de Paul Lazarsfeld y sus compañeros de la Escuela de Columbia (Katz \& Lazarsfeld, 
1955), cuando Lazarsfeld desarrolló su teoría de "the-two-step-flow of communication" que incidía en los efectos limitados de los medios. Además, Lazarsfeld indicó que la moda, por su dinamismo, era un campo propicio para el análisis del papel de la comunicación personal y del liderazgo de opinión. El presente artículo analiza los estudios en este campo entre los años 1950 y 2000; una evolución en la que se establecen las bases sobre las características de los líderes de opinión en moda como medidores de la cultura y valores sociales, fuentes de información, fuentes de consejo, modelos de comportamiento en moda, fuentes de seguridad para la aceptación social. La revisión cronológica de los estudios permite un análisis en profundidad en el que aparecen las claves sobre el papel de la comunicación personal, cuestión relevante en la difusión de las tendencias de moda.

Palabras clave: líderes de opinión, moda, difusión de tendencias, Lazarsfeld

\begin{abstract}
The studies about Opinion Leadership began with the contributions of Paul Lazarsfeld and his colleagues at the Columbia School (Katz \& Lazarsfeld, 1955), when Lazarsfeld developed his theory "The-two-Step-Flow of Communication", highlighting the limited effects of the media. Besides, Lazarsfeld showed that fashion was a favorable field for the analysis of the role of personal communication and opinion leadership. The present study analyzes the research in this field between the years 1950 and 2000; an evolution when the characteristics of fashion opinion leaders were settled. The chronological review of the studies provides an in-depth analysis in which the keys of the personal communication role in fashion innovations dissemination stand out.
\end{abstract}

Keywords: Opinion leadership; fashion; innovation diffusion; Lazarsfeld

\title{
Introducción
}

El auge de los influencers en el sector de la moda gracias a las redes sociales, primero con las blogueras y ahora con las instagramers, ha puesto en primera línea la cuestión de la influencia en la moda. Desde el ámbito profesional diversas revistas, consultores o empresas de comunicación han analizado el papel de los influencers en la comunicación y comercialización de productos de moda (Sammis et al., 2015; Schaefer, 2012; Solis \& Webber, 2012). Asimismo, múltiples académicos examinan los procesos de influencia en moda en el entorno online (Navarro \& Garcillán López-Rúa, 2016; Pedroni, Sádaba, \& SanMiguel, 2017; Sádaba \& SanMiguel, 2014; SanMiguel \& Sádaba, 2017; Wiedmann, Hennings, \& Langner, 2010).

Lejos de tratarse de una cuestión novedosa, el papel de la influencia es uno de los temas que han guiado los estudios sobre la moda desde la segunda mitad del siglo XX hasta nuestros días. Cambian los enfoques y las cuestiones analizadas, pero las 
preguntas sobre cómo se produce el proceso de influencia y quién influye realmente en este proceso han tenido una interesante evolución que es objeto de este artículo.

Los estudios en torno al poder de la comunicación personal, a través del liderazgo de opinión, se desarrollaron principalmente en el área de la opinión pública. A raíz del descubrimiento del impacto de los líderes de opinión, por parte de Paul Lazarsfeld y sus compañeros de la Escuela de Columbia, en The People's Choice (Lazarsfeld et al., 1944) y Personal Influence (Katz \& Lazarsfeld, 1955).

Lazarsfeld desarrolló una teoría sobre los efectos limitados de los medios, "the-twostep-flow of communication" (teoría del doble flujo), y expuso que los medios de masas no funcionan como herramientas omnipotentes para captar las audiencias y transmitir mensajes políticos y comerciales, sino que los mensajes de los medios son captados por un conjunto de individuos que transmiten la información al resto de la sociedad. Estos individuos serán denominados líderes de opinión. De este modo, mediante el análisis del flujo de la información entre los medios y las audiencias, se descubre que los medios no influían de forma directa sobre las personas. Esta idea era contraria a la planteada por Lasswell (1938), quién defendía que los medios de comunicación actuaban como una "aguja hipodérmica" impactando de forma directa sobre las audiencias y produciendo efectos inmediatos sobre éstas. En este caso, la opinión se forjaría a través de un esquema de influencia unilateral e intencionada (Wolf, 1987).

Las investigaciones realizadas por Lazarsfeld y sus seguidores (1955; 1944), demostraron cómo los individuos afirmaban que sus decisiones de voto o compra estaban más afectadas por sus relaciones personales que por los medios de comunicación. Señalaban que las principales fuentes de información e influencia eran sus grupos cotidianos, como la familia y los amigos. El análisis sobre la influencia personal lo desarrollaron en las áreas de la opinión pública, el consumo, la moda y acudir al cine.

Lazarsfeld y Katz (1955) identificaron como líderes de opinión o influentials a aquellas personas que dentro de grupos informales -amigos, familiares y vecinos-, cara a cara, guían la opinión y el cambio, ejerciendo un liderazgo "casi invisible, ciertamente inconsciente, íntimo e informal, de persona a persona, a través del contacto diario" (Katz \& Lazarsfeld, 2011, p. 140). En aquel primer estudio de 1955, Lazarsfeld y Katz prestaron atención al campo de la moda y analizaron como eran los líderes de opinión en moda.

¿Por qué Lazarsfeld decidió prestar atención a este sector? Él mismo nos da la respuesta en su investigación: "la moda es un sector dinámico propicio para la investigación, más que en el marketing u otras áreas de consumo, la moda es un escenario de constante cambio" (Katz \& Lazarsfeld, 2011: 249). Es la actividad de este 
sector, la dinámica de colecciones, temporadas y tendencias, lo que propicia la necesidad de líderes de opinión, que de forma ágil y atractiva se presentan como modelos de comportamiento en compra de moda para sus grupos.

No podemos olvidar, como señalaron Simmel (1923) o Lipovestki (1990), que la moda es el juego entre la diferenciación y la identificación; los individuos necesitan sentirse únicos y a la vez, parte del grupo. Esta realidad, este juego de fuerzas contrarias, dota de gran relevancia al papel de los líderes de opinión en la moda. El líder, no solo funciona como fuente de información, transmitiendo mensajes de los medios al grupo. El líder de opinión en moda muestra a sus pares qué es lo que se puede llevar o no, lo que está socialmente aceptado. Imitar al líder de opinión es eliminar el riesgo percibido al hacer una compra, y en moda, tener la garantía y respaldo del influyente elimina ese riesgo (Robertson, 1973). El precio de la adopción de una moda sin éxito puede ser la vergüenza social, y por el contrario, el éxito en una adopción tendrá como consecuencia la popularidad y la aceptación (Weimman, 1994). Los líderes de opinión ofrecen seguridad y apoyo social en la toma de decisiones en moda: ellos están actualizados, bien informados y perfectamente integrados en la sociedad.

Para las empresas de moda, el líder funciona como puerta de acceso al gran mercado. Conseguir que los líderes de opinión en moda apoyen una marca o producto significa la llave al mercado mayoritario. Según la curva de Rogers (1962), los líderes de opinión funcionan como innovadores y adoptantes tempranos, mostrando a la mayoría de forma temprana los productos que deben comprar.

Dada la importancia del papel de los influyentes en moda en la actualidad, la presente investigación tiene como objetivo asentar las bases teóricas sobre el papel de los líderes de opinión. Con este fin, se analizan los estudios sobre el liderazgo de opinión en moda desde 1955 hasta el 2000. Estos fueron realizados principalmente desde el área de la comunicación, la sociología y el marketing. A pesar de que Katz y Lazarsfeld (1955) afirmaran que la moda era un escenario propicio para el análisis del liderazgo de opinión, hay que puntualizar que, en comparación con otros campos de investigación, como la opinión pública o la política, los estudios en el campo de la moda fueron escasos. Sin embargo, son relevantes para poder entender las distintas aportaciones al campo de la influencia en moda, nos permiten comprender mejor cómo se produce este proceso y consiguen acercarnos también a la figura del influyente. 


\section{Metodología}

El estudio presenta la revisión bibliográfica sobre las investigaciones relacionadas con el liderazgo de opinión en moda. Se trata de un trabajo multidisciplinar; como se ha comentado anteriormente, estos estudios se realizaron desde las áreas de la comunicación, la sociología y el marketing. No todos los estudios presentan el mismo concepto de líderes de opinión que plantearon los investigadores de la Escuela de Columbia, pero todos ellos analizan o tienen en cuenta la importancia de actores que son innovadores, adoptantes tempranos o prescriptores del producto de moda.

Las diferentes investigaciones en el ámbito del liderazgo personal y el papel de los líderes en la difusión de tendencias de moda se presentan de forma cronológica, prestando atención a cada década y destacando las aportaciones más relevantes de cada estudio. Posteriormente, se añade un cuadro resumen que permite ver de forma clara y concisa la evolución de sus estudios y su enfoque.

Los diferentes estudios demuestran finalmente que la moda es una dualidad entre lo individual y lo colectivo, donde el circuito de la innovación y de la influencia de algunos genera la misma moda.

\section{Estudios sobre el liderazgo personal y la difusión de tendencias de moda (1950- 2000)}

\subsection{El descubrimiento de los líderes de opinión o influyentes}

Los estudios en torno al liderazgo de opinión comenzaron en la década de los 50 . Se desarrollaron principalmente en el área de la opinión pública, a raíz del descubrimiento del impacto de los líderes de opinión, por parte de Lazarsfeld y sus compañeros de la Escuela de Columbia, en el estudio de campañas presidenciales y la toma de decisiones por parte de los individuos a la hora de votar, investigación publicada en The People's Choice (Lazarsfeld, Berelson and Gaudet, 1944).

Posteriormente, Lazarsfeld y Katz (1955) explicaron en profundidad la teoría del doble flujo y el papel de los líderes de opinión en la transmisión de mensajes, en una investigación donde se analizó por primera vez a los influyentes en el área de la moda. 
Personal Influence fue el comienzo de una serie de estudios que analizaron el papel de los líderes de opinión en moda desde distintas perspectivas.

Aunque Personal Influence $(1955)^{1}$ fue el primer estudio que aplicó la teoría del doble flujo en el campo de la moda y destacó la importancia de la comunicación personal, sociólogos como Tarde o Simmel ya habían señalado el papel de la influencia en los procesos de trasmisión de modas. Ambos investigadores hablaron de la moda como un proceso de imitación, una "torre de agua de la que desciende una continua cascada de imitación" (Tarde, 1903: 221), donde las clases bajas quieren parecerse a las clases altas. Este proceso de imitación o contagio denota el deseo o aspiración de un grupo de individuos por parecerse a otros: son los individuos de la clase social baja influidos por los de la clase social alta.

Simmel, en su análisis sobre la moda realizado en 1905, señaló por primera vez la existencia de algunos individuos que van por delante del conjunto del grupo social liderado la adopción de nuevas modas (Simmel, 1957). Weimann apunta al respecto que ésta podría ser la primera descripción sobre el líder de moda y añade que Simmel no solo destaca la importancia de las figuras influyentes, sino que también señala como éstas se ajustan a las normas sociales, sirviendo de medidores de los cambios culturales y los valores sociales establecidos (Weimann, 1994: 146).

Una década antes de Personal Influence (1955), J.E Janney realizó un pequeño estudio en relación con el liderazgo de opinión en moda Fad and fashion leadership among undergraduate women (Janney, 1941). En dicho estudio no se contempla la teoría del doble flujo, ni se analizan en profundidad las características de los líderes de opinión en moda o cómo se produce la influencia; pero se destaca que las modas suelen ser originadas por mujeres jóvenes con prestigio en su grupo social, mujeres que además son líderes en otras áreas como el baile, el diseño o la decoración.

A mediados del siglo XX, Katz y Lazarsfeld (1955) precursores de las teorías sobre el papel de la influencia personal en la comunicación de masas, basaron su estudio en Decatur (EEUU) en las áreas de la política, el cine, el marketing y la moda. Los investigadores de la Escuela de Columbia prestaron atención al campo de la moda al ser un área de constante cambio y de comunicación continua, afirmando "más que en el marketing u otras áreas de consumo, la moda es un escenario de constante cambio. De hecho, la esencia de ser de 'estar a la moda' es la realización de cambios adecuados en el momento adecuado" (2011: 249). Katz y Lazarsfeld analizaron cuál era el interés de las mujeres de la muestra por la moda, cómo era la búsqueda de consejos y la relación de la moda con el ciclo de vida.

\footnotetext{
${ }^{1}$ La primera edición de Personal Influence es de 1955, en el presente artículo se utiliza la edición de 2011 para las citas textuales.
} 
El estudio realizado por Katz y Lazarsfeld realizó una serie de preguntas en relación con la moda: ¿Es para ti importante, moderadamente importante nada o nada importante estar a la moda? ¿Has realizado algún cambio para ir más a la moda? ¿Cuántos vestidos te has comprado o confeccionado en el último verano? El objetivo de estas preguntas era captar la importancia que tenía para cada mujer "estar a la moda" y en consecuencia, qué cambios había adoptado.

Katz y Lazarsfeld analizaron el flujo de influencia en moda. Señalaron que la moda, especialmente en el público femenino, despierta un alto interés que produce una demanda de liderazgo en los grupos, ya que el tráfico de consejos sobre moda es muy elevado, sobre todo en las chicas jóvenes (2011: 253). Personal Influence destaca la correlación existente entre el ciclo de vida de las encuestadas y su interés por la moda: existe más interés por la moda entre las mujeres jóvenes que entre las madres.

Personal Influence (2011) subraya la importancia de la sociabilidad en el liderazgo de moda; el estudio señala que el liderazgo en esta área se duplica entre aquellas personas que son altamente sociables. La sociabilidad no solo indica que las mujeres tengan un gran número de contactos, sino que tienen un gran interés por el tema, interaccionando con otras personas del mismo grupo y diferentes. Por otra parte, apunta que estas personas son sensibles a la impresión que causan en los otros y se preocupan por su interacción e integración entre los diferentes grupos y personas; actitud que se muestra al ir a la moda. En consecuencia, las mujeres que son más sociables tienen más oportunidades de ser preguntadas por consejos en moda, no solo porque están más accesibles para quienes buscan consejo, sino también porque están más preocupadas y activas en el mercado de la moda. Además, para liderar en moda no solo es necesario tener un alto interés y preocupación por la moda, el líder en moda debe ser buscado por otros individuos para obtener su consejo, ya que los individuos reconocen en él sus cualidades (2011: 259-260).

Katz y Lazarsfeld determinaron así que los líderes de opinión en moda se encontraban concentrados en las mujeres jóvenes, y dentro de este grupo, en aquellas que eran más sociables. Inciden en que el nivel o estado social no es el factor más importante para el liderazgo en moda, y que los líderes de opinión en moda se encuentran en todas las clases sociales y tienden a influir en su mismo status social; es decir, las mujeres líderes de opinión influyen a otras mujeres como ellas (2011: 269).

Dos años después de Personal Influence, Katz publica junto a Meyersohn Notes on a Natural History of Fads (1957), una investigación centrada en el campo de la moda en la que afirman: "las modas proporcionan una oportunidad extraordinaria para estudiar los procesos de influencia o contagio, del comportamiento innovador y cíclico, y del liderazgo (1957: 594). Meyersohn y Katz señalan que en los estudios de moda se ha dado un considerable interés, desde el punto de vista sociológico, a la cuestión de por qué un grupo en lugar de otro es el portador de ciertas modas, y apuntan que, en la 
mayoría de las sociedades, las mujeres son las agentes del cambio de modas en ropa (Meyersohn and Katz, 1957), como ya señalaron en Personal Influence (1955). Meyersohn y Katz exponen que también se han desarrollado algunos estudios que tratan de explicar los canales de información y consejo que afectan en las decisiones de los consumidores².

\subsection{Los influyentes como difusores de la innovación y agentes de cambio}

La siguiente década en los estudios de influencia y moda comenzó con la aportación de Rogers (1962) y su estudio sobre la difusión de innovaciones. Rogers señala que los individuos buscan información con el fin de disminuir la incertidumbre y el riesgo percibido ante una nueva innovación. Esta información la buscan principalmente en los canales de comunicación personal, a través del cara a cara con otros individuos, canal más eficaz para la formación o cambio de actitudes y que influye en la decisión de adoptar o rechazar una nueva idea o producto. Rogers puntualiza que los individuos evalúan una innovación a través de las valoraciones subjetivas de compañeros cercanos que han adoptado esa innovación.

Las aportaciones de Rogers (1962), en línea con lo revelado por Katz y Lazarsfeld (1955) en torno a la influencia personal, destacan que algunos individuos que adoptan innovaciones antes que otros ejercen su liderazgo de opinión al servir de modelo para sus seguidores: son fuentes de información fiable y consejo, e influyen en las actitudes de otros de un modo informal. Estos individuos ejercen como influyentes especialmente en la etapa de decisión de adopción de innovaciones y entre individuos semejantes a ellos -con similar educación, condición social o creencias-, como también destacó Personal Influence (1955).

A raíz del descubrimiento del papel de la influencia personal en la transmisión de mensajes o innovaciones a las masas, King (1965) en su análisis sobre la difusión de modas -Fashion adoption: A rebuttal to the'trickle down'theory-, destaca el papel de los líderes de opinión en moda, existentes en cada clase social, como fuente de información y difusión de nuevas modas.

Tras las aportaciones de los investigadores de la Escuela de Columbia, Anspach (1967) en The way of fashion analizó a los influyentes en moda y desarrolló un concepto que se alejaba de los parámetros establecidos por Lazarsfeld y sus colegas. Para Anspach, los innovadores en el mundo de la moda no suelen ser los líderes de opinión. Los

\footnotetext{
${ }^{2}$ Meyersohn y Katz se refieren al estudio de Personal Influence (1955) en el que explican que las mujeres buscan consejo e información en otras mujeres que detectan como líderes de opinión dentro de su mismo grupo social.
} 
innovadores son aquellos que adoptan por primera vez una nueva moda introduciendo así una "nueva chispa" que dará lugar a un cambio, este pequeño cambio es el que los líderes de opinión deben de seguir y difundir en la sociedad.

De este modo, Anspach señala que el líder de opinión es quien difunde las nuevas modas, y propone una subdivisión en las categorías de líderes de opinión. En primer lugar, estarían los líderes de opinión de la comunidad, las celebrities, las cuales adoptan antes las nuevas modas y las exponen a las masas. En segundo lugar, estarían los líderes locales, individuos accesibles a toda la comunidad, encargados de juzgar, apoyar o desacreditar la nueva moda que los primeros han propuesto. El primer tipo de líderes propuesto por Anspach se aleja de la definición anterior de líder, pues son personas que gozan de un prestigio social y por ello una posición de liderazgo, no influyen a través del contacto personal, cara a cara, cotidiano, íntimo e informal de los influentials propuestos en 1955 por Katz y Lazarsfeld.

Hay que tener en cuenta que, en moda, la imitación de otros individuos juega un papel fundamental. Por lo general, el individuo quiere rechazar cualquier tipo de riesgo, de vergüenza o exclusión social, debido a la adopción de una moda no adecuada. Por ello, la idea de Anspach respecto al liderazgo de opinión en moda, muestra que posiblemente en este campo, como Weimann apunta (1994: 146), el proceso de influencia personal puede implicar un flujo de dos pasos dentro de los líderes: primero de los líderes de la comunidad a los líderes locales, y segundo, de los líderes locales a sus amigos y conocidos. Los primeros ejercen su influencia como modelos a seguir y ser imitados; y los segundos como individuos a los que pedir consejo para la toma de decisiones.

Margaret Grindereng, en Fashion Diffusion (1967) analizó el proceso de difusión de las modas y sugirió el papel de los "agentes del cambio" -influyentes, innovadores o consumidores conscientes de la moda- que sirven de ejemplo a otros consumidores, los cuales visualizan en estos agentes los nuevos estilos, y son fuente de influencia verbal para la toma de decisiones.

Blumer destaca también el papel de los líderes de opinión en el flujo de la moda (Blumer, 1969). Señala la existencia de individuos que funcionan como figuras de prestigio y que presentan modelos de comportamiento; individuos que gozan de tal reputación que el resto de grupo reconoce que están calificados para juzgar el valor o la idoneidad de una moda, siendo su elección una garantía o aval de superioridad o decoro para el resto de individuos. La combinación de tales figuras prestigiosas, que exponen el mismo modelo, aumenta la probabilidad de adopción (Blumer, 1969). Blumer presta especial atención al carácter cambiante de la moda y su orientación subjetiva mediada por el gusto - "taste"- de los líderes. Del trabajo de Blumer se desprende el concepto de los "tastemakers" individuos difusores de tendencias que actúan como intermediarios e interpretan la moda para el público general. Este 
concepto fue analizado en profundidad por investigadores como Bourdieu (2002) y, en el campo de la moda por Matthews (2015).

\subsection{Las características y tipos de influyentes}

Una década más tarde de la aportación de Rogers, John Summers realizó el primer estudio centrado en el liderazgo de opinión en moda bajo el título The identity of women's clothing fashion opinion leaders (Summers, 1970). Basándose en los descubrimientos de Lazarsfeld y Katz, Summers define el perfil de los influyentes en moda, a través de tres grupos de características: demográficas, sociales y actitudinales. Para Summers, los líderes de opinión en moda ejercían un papel fundamental en la difusión de nuevas modas, ya que, con el apoyo de los líderes, éstas tienen una mayor visibilidad, resultado de su participación activa en moda y su extensa actividad social.

William Darden y Fred Reynolds (1972), realizaron otro de los estudios focalizados en el liderazgo de opinión en moda, esta vez centrado en el público masculino. Para Darden y Reynolds, los líderes de opinión eran aquellos individuos que tienden a ser transmisores interpersonales de información e influencia en un mayor grado que el resto de personas de un sistema social (1972: 324). En su estudio, realizan una aplicación de las escalas de medición de líderes de opinión por el método de autodesignación, desarrolladas por Rogers (1962) y modificadas por King y Summers (1970).

Además, Darden y Reynolds presentaron un análisis de 17 variables independientes para determinar el perfil de los influyentes en moda (1972: 325). Estas variables se dividían en tres grupos de características: las que predisponen al liderazgo, las demográficas y las sociales. En las características que predisponen al liderazgo analizaron: el interés por la moda, la autoconfianza generalizada, la búsqueda de información, la actitud hacia el cambio, la claridad cognitiva, el estilo cognitivo, la conciencia de color (la preocupación por los colores y su combinación), la confianza relativa en sí mismo, el atrevimiento en moda, la popularidad relativa y la percepción de riesgo. Los investigadores concluyen que algunas variables predicen de forma más clara el liderazgo en moda, como el interés por la moda y el atrevimiento en moda.

Schrank y Gilmore (1973) plantearon una nueva escala para identificar a los líderes de opinión en moda. Dicha escala contaba con tres apartados -liderazgo de opinión, actitud hacia la conformidad e interés en la ropa- y 20 variables cada uno, con el fin de analizar y relacionar los comportamientos en el campo de la moda. En su estudio, además, observaron las funciones que realizan dichos líderes; para ello examinaron la innovación en moda relacionada con aquellos individuos que realizan una adquisición anticipada de productos de tendencias y el uso de los nuevos artículos de moda. 
Algunas de las conclusiones que obtuvieron Schrank y Gilmore (1973) fueron que existe una relación entre el liderazgo de opinión en moda y la innovación en la adopción de nuevas prendas o estilos; que algunos individuos juegan un doble papel adoptando cambios e influyendo verbalmente en los demás para que adopten esos cambios; que existen diferencias palpables entre el papel que tienen los líderes visuales o verbales respecto a los no líderes; que los líderes de opinión y los innovadores suelen ser las mismas personas, o que las variables asociadas al liderazgo de opinión o innovación difieren ligeramente. Los autores puntualizaron que la relación positiva entre liderazgo de opinión e innovación en moda se puede deber a "la velocidad con que las innovaciones en ropa se difunden en comparación con la mayoría de otras innovaciones de adopción rápida, sobre todo entre las mujeres universitarias" (1973: 539). Asimismo, expusieron que la influencia en moda debe ser ejercida de forma rápida para que el proceso de adopción sea efectivo y que "el número de personas disponibles para influir disminuye a medida que aumenta la adopción, por lo que tal vez la adopción temprana de una innovación añade validez a la función de un líder de opinión” (1973: 539).

George Sproles y Charles King (1973) desarrollaron una investigación en torno al consumidor como agente del cambio. Los autores señalan que algunos consumidores actúan como líderes de opinión promoviendo cambios entre sus pares. Posteriormente Sproles, en su explicación del proceso de la moda en Fashion Theory (1974), vuelve a destacar el papel relevante de los líderes de moda como agentes de cambio y de la comunicación personal en la difusión de modas. Sproles señala a los líderes de moda como un pequeño grupo de consumidores más conscientes de la moda y sus innovaciones, que adoptan e introducen nuevas tendencias o productos para sus seguidores. Afirma que el concepto "agentes del cambio" en moda es equiparable al del líder de opinión y que estos individuos, al seguir más de cerca las nuevas tendencias y estar mejor informados, dan a conocer las tendencias a sus seguidores e influyen en ellos a la hora de adoptarlas o rechazarlas. Sproles no analizó las características de estos líderes ni desarrolló ningún tipo de escala de medición; se limitó a destacar el papel de estos individuos en la difusión de modas.

Joyce Brett y Anne Kernaleguen (1975) analizan las características que diferencian a los líderes de opinión en moda de los no líderes y proponen la identificación de los líderes de moda a través del análisis de su personalidad ${ }^{3}$.

Por su parte, Steven Baumgarten (1975) explica la relación entre los innovadores en moda y los líderes de opinión. Baumgarten afirma que la moda es un campo propicio

\footnotetext{
${ }^{3}$ El estudio de Brett y Kernaleguen (1975) fue realizado considerando la escala de medición propuesta por Rogers (1962). Es un estudio de corta extensión que no aporta muchos datos respecto a la personalidad de los líderes de opinión. La variable que analizan es la ansiedad respecto a los cambios en moda, destacando que los líderes presentaban un nivel de ansiedad más bajo.
} 
para el estudio de las innovaciones, ya que frecuentemente se introducen nuevas ropas y estilos en el mercado. Ante la dicotomía que plantea Rogers (1962) entre innovadores y líderes de opinión, Baumgarten confirma la existencia de un grupo de individuos, agentes del cambio, que serían innovadores y líderes de opinión al mismo tiempo, individuos que denominaría "comunicadores innovadores" los cuales adoptarían tendencias antes que el resto del grupo y ejercerían un rol de influyentes, informando y aconsejado sobre estas tendencias.

Los investigadores Douglas Tiger, Lawrence Ring y Charles King desarrollaron varias investigaciones en el campo de la moda en Canadá, en las que analizaron la participación en moda y el comportamiento del consumidor. En 1975 en Contemporary Fashion Theory and Retail Shopping Behavior: A Segmentation Analysis, analizan la participación en moda -fashion involvement- basándose en las cinco dimensiones de los consumidores de moda desarrolladas por Sproles y King (1973): la innovación a la hora de la compra, la comunicación interpersonal, el interés, el conocimiento, la conciencia en moda y la reacción ante los cambios de modas. Con estas dimensiones Tiger, Ring y King (1975) establecen un índice de participación en moda. Un año más tarde, en Fashion Involvement and Buying Behavior: a Methodological Study (Tigert, Ring and King, 1976), continúan su investigación sobre la participación en moda y explican que la participación responde a un conjunto de factores que introducen los consumidores en su relación con la moda.

Tiger, Ring y King (1975) detallan que la investigación en moda se ha centrado en dos campos relevantes para las ventas y el volumen del mercado: los innovadores o early adopters de las nuevas tendencias o estilos; y los líderes de opinión de la moda o comunicadores interpersonales, quienes legitiman -en grupo o de forma personal- los nuevos conceptos de ropa, idea señalada y ya por Rogers (1962).

En este sentido, Elizabeth Hirschman y William Adcock, realizaron un análisis sobre los comunicadores innovadores en moda en 1978. A raíz del concepto desarrollado por Baumgarten (1975), elaboraron una investigación en la moda masculina con el fin de analizar cuatro categorías de individuos: comunicadores innovadores, innovadores, líderes de opinión o el conjunto de la población. Las variables que utilizan para comparar las categorías de individuos son: las características socioeconómicas; influencias sociográficas; evaluaciones de atributos de la tienda; uso de medios de comunicación; y los patrocinios en tienda.

\subsection{El influyente en los consumidores de moda}

A comienzos de la década de los 80, Tiger, King y Ring publicaron otro estudio sobre la participación en moda (1980). En este caso, analizaron la participación en moda y los agentes del cambio en cuatro escenarios culturales diferentes; investigando las 
reacciones a las modas cambiantes, el "awareness" en moda, el conocimiento, los estilos de vida y el comportamiento de compra.

Sproles (1981) realiza una minuciosa investigación sobre los ciclos de vida del producto en moda. Explica cómo el análisis de estos ciclos ayuda a comprender mejor los fenómenos del marketing y los procesos de elección de los consumidores, ya que "las modas son, por definición, los fenómenos cíclicos temporales adoptadas por los consumidores durante un tiempo y situación particular" (Sproles, 1981: 116).

Sproles presta atención al papel de los líderes de moda como consumidores, en las diferentes etapas del ciclo de vida del producto ${ }^{4}$, y destaca que Katz y Lazarsfeld (1955) propusieron que el líder de la moda más importante "no es necesariamente una persona encantadora, pero anónima ${ }^{5}$, mostrando estilos caros. Más bien, sugieren que la influencia de la moda se produce de manera informal entre amigos personales que interactúan en los círculos sociales similares" (Sproles, 1981: 118).

Señala este autor que no solo la industria es propagadora de nuevas modas ${ }^{6}$, sino que el propio comportamiento del consumidor también lo hace, debido a la gran variedad de estilos disponibles y a la relajación de las normas sociales a la hora de vestir, que permite a los consumidores tomar sus propias elecciones y crear sus propios estilos: "Como alternativa al liderazgo industrial en tendencias, se apunta a los consumidores como los principales iniciadores y propagadores de lo que va a ponerse de moda" (Sproles, 1981: 118). De este modo, la moda pasaría de introducirse desde las marcas a los consumidores a realizar el camino inverso, desde "abajo" hacia "arriba".

Por otro lado, Leslie Davis y Lennon Sharron (1985) señalaron que los líderes de opinión tienden a tener más auto-monitorización que los no líderes. Afirmaron que las mujeres líderes de opinión en moda tenían un mayor índice de auto-monitorización que influye en su consumo y participación en moda. Davis y Sharron apuntan que los líderes de opinión en moda usan la ropa para lograr la aprobación social, motivados por su utilidad en la transmisión de mensajes apropiados para diferentes situaciones sociales, es decir, la ropa es el modo de expresar su auto-concepto, la autoestima y la auto-percepción.

\footnotetext{
4 “Introducción y adopción por parte de los líderes de la moda, el aumento de la aceptación del público (crecimiento), la conformidad de masas (maduración), y el inevitable declive y obsolescencia esperando todas las modas" (Sproles, 1981, p. 116).

${ }^{5}$ Sproles identifica 'anónima' con una persona que, aunque popularmente se puede saber quién es no se la puede conocer de manera personal.

${ }^{6}$ Sproles (1981: 118) señala que históricamente la industria de la moda ejercía un poderoso control, casi dictatorial, en la difusión de tendencias de moda. Poder que se debida al papel de los diseñadores de alta costura en Europa y Estados Unidos, al influjo de la publicidad y de medios de comunicación de la industria de la moda como Womens Wear Daily.
} 


\subsection{Influyentes y valores sociales}

A principios de los años 90, Ronald Goldsmith y sus colegas desarrollaron tres estudios en relación con los valores sociales, la moda y el liderazgo o la innovación: Social Values and Fashion Leadership (1991), Social values and female fashion leadership: A cross-cultural study (1993) and Social Values and Fashion Innovators (1993).

Goldsmith, Freiden y Kilsheimer (1993) examinaron la importancia relativa asignada a los valores sociales por parte de los líderes de moda femenina en comparación con los no líderes, en un estudio realizado anteriormente en 1991, pero al cual incorporan la comparación entre Estados Unidos y Reino Unido. Primero, identificaron a los líderes de opinión en moda a través de una escala de autoevaluación y posteriormente, evaluaron los valores sociales. Goldsmith et al. señalan que el liderazgo en moda "es un concepto importante en la comercialización debido a que el líder de la moda juega un papel clave en la difusión de nuevas modas" (1993: 402). Recalcan que los líderes en moda aprenden sobre nuevas tendencias de la moda y adquieren nuevos productos recién introducidos en el mercado antes que el resto de consumidores, y son esenciales en la propagación de nuevas modas.

Los investigadores explican que los valores "juegan un papel importante en el consumo de prendas de vestir, sobre todo en las nuevas modas, porque la ropa debe satisfacer las necesidades expresivas y simbólicas para los consumidores" (1993: 405). Analizaron valores sociales como: la pertenencia, la emoción, la diversión, las relaciones cálidas, la autorrealización o la seguridad. Concluyeron que el valor de la emoción es el más destacado por los líderes de opinión, por lo que recomendaron que éste fuera incorporado en la publicidad y las promociones dirigidas a los líderes de la moda. Goldsmith y Stith (1993) descubrieron que los innovadores daban más importancia a la emoción y a la diversión, al igual que los líderes de opinión, por lo que la moda estaría positivamente relacionada con estos valores.

Durante el mismo año, Jane Workman y Kim Johnson (1993) analizaron el liderazgo de opinión y la innovación en moda. Los resultados de este estudio sugieren que una de las motivaciones detrás de la adopción y difusión de nuevos estilos es una necesidad de variedad en los cambios de ropa, estímulo que mueve por igual tanto a los innovadores como a los líderes de opinión, y en mayor medida que a sus seguidores.

Teniendo en cuenta el papel de los medios de comunicación, Christopher Miller, Shelby Mclntyre y Murali Mantrala (1993) destacaron el papel de la influencia selectiva en el proceso de la moda. Los investigadores exponen que los individuos interactúan, de forma verbal o no, con otras personas para aprender el significado de una nueva moda y saber si la adopción de esta es apropiada o no. Miller et al. (1993) hablan de influencia selectiva en moda, dado que un influyente puede influir a algunos individuos y a otros no, es decir no todos los líderes en moda influyen en todo su círculo social y tampoco lo hacen del mismo modo. Toward Formalizing Fashion Theory señala la 
complejidad del estudio de los patrones de influencia, donde algunos medios de comunicación permiten a ciertos individuos ejercer una amplia influencia en la sociedad. El estudio matiza que, a pesar de la amplia cobertura de los medios de comunicación, los patrones de influencia no se pueden homogeneizar por completo y los medios de comunicación no pueden crear individuos con una capacidad de influencia suficiente como para dictar exclusivamente modas y tendencias.

Gabriele Weimann, en su libro The influentials: People who influence people (1994), en el que realizó una revisión de los estudios en torno al liderazgo de opinión, al igual que Katz y Lazarsfeld (1955), dedicó un capítulo a los líderes de opinión en moda, en el que concluye que los influyentes en moda tienen gran importancia en la difusión y adopción de tendencias de moda en la sociedad.

Finalmente, cabe destacar el estudio realizado por Beaudoin, Moore y Goldsmith a finales de los años 90, Young fashion leaders' and followers' attitudes toward American and imported apparel (1998). Baudoin et al. afirman que:

...los líderes en moda pueden ser definidos como aquellos que están más interesados en la moda que otros consumidores en el mercado, que son más seguros de su propio gusto, que son los primeros en adquirir nuevos estilos $y$, sobre todo, que influyen otros consumidores a adoptar y comprar nuevos artículos de moda (1998: 194).

En su estudio definen las características de los líderes de opinión en moda en América y analizan la actitud de estos individuos frente a las prendas de vestir importadas y nacionales, a través de la evaluación de 12 atributos como el buen gusto, la durabilidad, el precio, la comodidad, el color o la calidad. Concluyen que los líderes de opinión tienen mejor actitud hacia la ropa importada que los no líderes y un papel clave para la difusión de ropa, tanto en marcas nacionales como internacionales.

Tabla 1: Resumen de los principales estudios en torno a los líderes de opinión en moda 1950-2000

\begin{tabular}{|c|c|c|c|}
\hline Publicación & Autores & $\begin{array}{l}\text { Año de } \\
\text { publicación }\end{array}$ & Aportaciones \\
\hline $\begin{array}{l}\text { Fad and fashion } \\
\text { leadership among } \\
\text { undergraduate } \\
\text { women }\end{array}$ & Janney & 1941 & $\begin{array}{l}\text { Modas suelen ser originadas por mujeres } \\
\text { jóvenes con prestigio en su grupo social. Líderes } \\
\text { también en otras áreas como el baile, el diseño } \\
\text { o la decoración. }\end{array}$ \\
\hline Personal Influence & $\begin{array}{l}\text { Katz y } \\
\text { Lazarsfeld }\end{array}$ & 1955 & $\begin{array}{l}\text { 'Estar a la moda' es la realización de cambios } \\
\text { adecuados en el momento adecuado. } \\
\text { Tráfico de consejos sobre moda es muy } \\
\text { elevado, especialmente en las chicas jóvenes } \\
\text { Mujeres jóvenes eran a menudo más }\end{array}$ \\
\hline
\end{tabular}


Notes on a Natural

Meyersohn

1957 History of Fads

y Katz

Rogers

1962

innovations

Fashion adoption: A

King

rebuttal to the'trickle down'theory

The way of fashion

Anspach

Fashion Diffusion

Grindereng

Fashion: From class differentiation to collective selection

The identity of women's clothing fashion opinion leaders

$\begin{array}{ll}\begin{array}{l}\text { Predicting opinion } \\ \text { leadership for men's } \\ \text { apparel fashions }\end{array} & \begin{array}{l}\text { Darden y } \\ \text { Reynolds }\end{array} \\ \begin{array}{l}\text { Correlates of Fashion } \\ \text { Leadership: }\end{array} & \begin{array}{l}\text { Schrank y } \\ \text { Implications for }\end{array} \\ \begin{array}{l}\text { Fashion Process } \\ \text { Theory }\end{array} \\ \begin{array}{l}\text { The Consumer Fashion } \\ \text { Change Agent: } A\end{array} \\ \begin{array}{l}\text { Theoretical } \\ \text { Conceptualization and }\end{array} \\ \begin{array}{l}\text { Empirical } \\ \text { King }\end{array}\end{array}$

influyentes. Son sociables, con gran número de contactos, sensibles a la impresión que causan.

Señalan que la moda proporciona una oportunidad extraordinaria para estudiar los procesos de influencia o contagio, del comportamiento innovador y cíclico, y del liderazgo.

La comunicación interpersonal es buscada por los individuos para disminuir la incertidumbre y el riesgo percibido ante una nueva innovación.

Destaca que los líderes de opinión en moda se encuentran en todas las clases sociales.

Diferencia entre líderes e innovadores. Señala que los primeros en adoptar modas son las celebridades y después líderes de opinión que difunden las modas a las masas.

Explica el papel de los agentes del cambio en moda, fuentes de influencia verbal para la toma de decisiones de los consumidores.

Los líderes son figuras de prestigio y que presentan modelos de comportamiento, gozan de tal prestigio, están calificados para juzgar el valor o la idoneidad de una moda, siendo su elección una garantía o aval de superioridad.

Análisis de las características demográficas, sociales y actitudinales de los líderes de opinión en moda.

Análisis de los líderes de opinión a través del método de auto-designación. Propuesta de 17 variables del perfil de los influyentes en moda.

Nueva escala de medición del liderazgo en moda. Analizan la actitud hacia la conformidad y el interés por la ropa. Señalan que algunos individuos juegan un doble papel de líderes e innovadores.

Desarrollan cinco dimensiones que afectan a los consumidores como agentes del cambio 
Identification

Fashion Theory

Sproles

1975

Perceptual and personality variables related to opinion leadership in fashion

The Innovative Communicator in the Diffusion Process

Contemporary Fashion Theory and Retail Shopping Behavior: A Segmentation Analysis

Fashion Involvement and Buying Behavior: a Methodological Study

An examination of innovative communicator opinion leaders and innovators for men fashion apparel

Fashion involvement a cross-cultural comparative analysis

Analyzing fashion life cycles: principles and perspectives

$\begin{array}{lll}\begin{array}{l}\text { Self-monitoring, } \\ \text { fashion opinion } \\ \text { leadership, and } \\ \text { attitudes toward } \\ \text { clothing }\end{array} & \begin{array}{l}\text { Davis y } \\ \text { Sharron }\end{array} & 1985 \\ \begin{array}{l}\text { Social Values and } \\ \text { Fashion Leadership }\end{array} & \begin{array}{l}\text { Goldsmith, } \\ \text { Heitmeyer y } \\ \text { Freiden }\end{array} & \\ & 1991 \\ \begin{array}{l}\text { Social values and } \\ \text { female fashion } \\ \text { leadership: } \text { A cross- } \\ \text { cultural study }\end{array} & \begin{array}{l}\text { Goldsmith, } \\ \text { Freiden y }\end{array} & \\ \text { Kilsheimer } & \\ \text { Social Values and } & \text { Goldsmith y } & 1993\end{array}$

Tiger, Ring y 1976 King

Hirschman y 1978 Adcock

Tiger, Ring y 1980 King

Sproles
Explica el proceso de la moda. Señala a los líderes en moda como agentes del cambio y difusores de modas.

Analiza las características y la personalidad de los líderes en moda

Señala la idoneidad del campo de la moda para el estudio de las innovaciones. Expone el perfil del comunicador innovador.

Analizan la participación en moda como característica del liderazgo.

Desarrollan una lista de 24 factores que miden los estilos de vida y la participación en moda

Investigan la moda masculina y analizan cuatro categorías de individuos: comunicadores innovadores, innovadores, líderes de opinión o el conjunto de la población.

Analizan la participación en moda, la conciencia en moda y los estilos de vida.

Estudia el ciclo de vida de los productos de moda y el papel de los líderes de opinión en cada etapa.

Explican la auto-monitorización como característica relevante de los líderes.

Analizan los valores sociales y su relación con los líderes de opinión en EEUU.

Analizan los valores sociales y su relación con los líderes de opinión a través de un análisis comparativo EEUU y Reino Unido. Destacan el papel clave del líder en la difusión de modas.

Señalan las diferencias entre los líderes y los 


\begin{tabular}{|c|c|c|c|}
\hline Fashion Innovators & Stith & & innovadores y los valores sociales de cada uno. \\
\hline $\begin{array}{l}\text { Fashion opinion } \\
\text { leadership, fashion } \\
\text { innovativeness, and } \\
\text { need for variety }\end{array}$ & $\begin{array}{l}\text { Workman y } \\
\text { Johnson }\end{array}$ & 1993 & $\begin{array}{l}\text { Señalan que la necesidad variar en los cambios } \\
\text { de ropa es lo que mueve a la adopción y } \\
\text { difusión. }\end{array}$ \\
\hline $\begin{array}{l}\text { Toward Formalizing } \\
\text { Fashion Theory }\end{array}$ & $\begin{array}{l}\text { Miller, } \\
\text { Mclntyre y } \\
\text { Mantrala }\end{array}$ & 1993 & $\begin{array}{l}\text { Señalan la complejidad de los patrones de } \\
\text { influencia en moda. }\end{array}$ \\
\hline $\begin{array}{l}\text { The influentials: } \\
\text { People who influence } \\
\text { people }\end{array}$ & Weimann & 1994 & $\begin{array}{l}\text { Destaca el papel de los líderes en la difusión y } \\
\text { adopción de nuevas modas. }\end{array}$ \\
\hline $\begin{array}{l}\text { Young fashion leaders' } \\
\text { and followers' } \\
\text { attitudes toward } \\
\text { American and } \\
\text { imported apparel }\end{array}$ & $\begin{array}{l}\text { Beaudoin, } \\
\text { Moore y } \\
\text { Goldsmith }\end{array}$ & 1998 & $\begin{array}{l}\text { Definen las características de los líderes de } \\
\text { opinión en moda en América y analizan la } \\
\text { actitud de estos individuos frente a las prendas } \\
\text { de vestir importadas y nacionales, a través de la } \\
\text { evaluación de } 12 \text { atributos. }\end{array}$ \\
\hline
\end{tabular}

\section{Conclusiones}

A lo largo de los años, se ha prestado especial atención a los mass media como agentes socializadores en el campo de la moda. Las revistas de moda eran las encargadas de mostrar al gran público cuáles eran las nuevas tendencias, cómo llevarlas y dónde comprarlas.

Las aportaciones de Lazarsfeld y los investigadores de la Escuela de Columbia dieron lugar a un cambio de paradigma, pasando de una concepción de los medios de comunicación de masas con efectos todopoderosos, que impactaban de forma directa en las audiencias, a una visión que destacaba el efecto limitado de los medios y el papel de los líderes de opinión: individuos que captan mensajes -tendencias o nuevos productos- y los difunden entre sus círculos cercanos.

Tras el análisis desarrollado, se puede afirmar que los influyentes ejercen un papel relevante en el proceso de difusión y adopción de modas. De este modo, funcionan como: medidores de la cultura y valores sociales (Simmel, 1993; Katz y Lazarsfeld, 1955); fuentes de información (Katz y Lazarsfeld, 1955; Rogers, 1962; Blumer, 1969; Goldsmith, 1993); fuentes de consejo (Katz y Lazarsfeld, 1955; Rogers, 1962; Baumgarten, 1975; Goldsmith, 1993 ); modelos de comportamiento en moda (Katz y Lazarsfeld, 1955; Anspach, 1967; Beaudoin 1998); fuentes de seguridad para la aceptación social (Simmel, 1903; Rogers, 1962); o difusores de innovaciones (Rogers, 
1962; King, 1963; Anspach, 1967; Grindereng, 1967; Blummer, 1969; Gilmore, 1973; Sproles, 1973; Baumgarten, 1975; Goldsmith, 1993).

"En el caso de la moda, más que en cualquier otra área, la adopción de un nuevo artículo de moda se basa en gran medida en la aceptación social percibida" (Weimman, 1994: 156). En este sentido, lo influyentes ejercerían un papel crucial, pues "nadie mejor que los líderes de opinión actualizados, bien informados, y socialmente integrados puede proporcionar el apoyo social necesario y el respaldo" (1994: 156) para las nuevas modas. Idea que han respaldado los estudios analizados al encontrar en los líderes de opinión en moda la fuente de influencia más eficaz para la adopción de nuevos productos o tendencias por parte de los consumidores.

Los líderes de opinión en moda funcionan como ejemplos de comportamiento para los demás individuos (Grindereng, 1967), se convierte en fuentes de asesoramiento, y son garantía y respaldo en la moda seleccionada (Weimman, 1994). Los individuos necesitan saber que aciertan en el uso de un nuevo artículo de moda, pues en este campo especialmente quieren conseguir la aceptación social; por ello, quieren estar al tanto, a través de sus fuentes de información de confianza, de todos los cambios en las tendencias en moda y estilos de vida.

La moda es el reflejo de la sociedad, un proceso de imitación y diferenciación mediante el cual las personas pueden construir y comunicar su identidad. Por ello, la moda se entiende como un mecanismo social de conducta colectiva. Como consecuencia, los líderes de opinión en moda no son solo un grupo de individuos con un gran interés por la moda que funcionan como fuentes de información y consejo. El liderazgo en moda también comprende una participación activa en la moda y los estilos de vida; es decir, la adopción de cambios constantes antes que la mayoría de los individuos. Los líderes de opinión en este sector actúan como democratizadores de la moda, al dar a conocer de forma rápida y eficaz, y en todas las clases sociales, los estilos y tendencias imperantes en cada momento (King, 1963).

La presente investigación pone de relieve el papel de los líderes de opinión en el flujo de comunicación de información sobre moda. Durante la segunda mitad del siglo XX, los influyentes o influentials -entendidos como individuos que dentro de círculos cercanos, ejercen un liderazgo informal, de persona apersona, a través del contacto diario-, han funcionado como altavoces y difusores de los mensajes de las marcas de moda y mass media; y, al mismo tiempo, han guiado la opinión y el comportamiento de sus grupos.

La generalización del uso de internet y el auge de las redes sociales ha propiciado un incremento exponencial de líderes de opinión en la red, conocidos como influencers; blogueros, instagramers o youtubers que conviven y colaboran con los medios de comunicación tradicionales. Estudios realizados en los últimos años, analizan a los 
influencers o influentials en la era digital (Curiel \& Ortiz, 2018; Paço \& Oliveira, 2017; Sádaba \& SanMiguel, 2014; SanMiguel \& Sádaba, 2017).

Futuras investigaciones deberán profundizar en el impacto de los influencers en la difusión de nuevas tendencias y productos de moda, así como en el impacto en el comportamiento del consumidor. Asimismo, será conveniente analizar la evolución del liderazgo de opinión, características y conceptos en el entorno online.

\section{Referencias bibliográficas}

BAUMGARTEN, S.A. (1975): "The innovative communicator in the diffusion process". Journal of Marketing Research, JSTOR, 12(1), pp. 12-18.

BEAUDOIN, P., MOORE, M.A., AND GOLDSMITH, R.E. (1998): "Young fashion leaders' and followers' attitudes toward American and imported apparel". Journal of Product \& Brand Management, MCB UP Ltd, 7(3), p. 193.

BLUMER, H. (1969): "Fashion: From Class Differentiation to Collective Selection". The Sociological Quarterly. Wiley on behalf of the Midwest Sociological Society, 10(3), pp. 275-291.

BOURDIEU, P. (2002): La distinción: criterio y bases sociales del gusto. México: Taurus.

BRETT, J.E. AND KERNALEGUEN, A. (1975): "Perceptual and personality variables related to opinion leadership in fashion". Perceptual and motor skills. Perceptual \& Motor Skills, 40(3), pp. 775-779.

CURIEL, C. P., AND ORTIZ, S. L. (2018): “El marketing de influencia en moda. Estudio del nuevo modelo de consumo en Instagram de los millennials universitarios". adComunica, (15), 255-281.

DARDEN, W.R. AND REYNOLDS, F.D. (1972): “Predicting opinion leadership for men's apparel fashions," Journal of Marketing Research. JSTOR, 9(3), pp. 324-328.

DAVIS, L.L. AND LENNON, S.J. (1985): "Self-monitoring, fashion opinion leadership, and attitudes toward clothing," Psychology of Fashion. Health. Lexington, MA, pp. 177-182.

GOLDSMITH, R.E. AND STITH, T. (1993): "The Social Value of Fashion Innovators". Journal of Applied Bussiness Research, (9)1, pp. 10-16. 
GOLDSMITH, R.E., FREIDEN, J.B. AND KILSHEIMER, J.C. (1993): "Social values and female fashion leadership: A cross-cultural study". Psychology \& Marketing. Wiley Online Library, 10(5), pp. 399-412.

GOLDSMITH, R.E., HEITMEYER, J. R. AND FREIDEN, J.B. (1991): "Social values and fashion leadership". Clothing and Textiles Research Journal. Sage Publications, 10(1), pp. 37-45.

GRINDERENG, M.P. (1967): "Fashion diffusion". Journal of Home Economics, 59(3), pp. 171-174.

JANNEY, J.E. (1941): "Fad and fashion leadership among undergraduate women". The Journal of Abnormal and Social Psychology. American Psychological Association, 36(2), p. 275.

KATZ, E. AND LAZARSFELD, P.F. (1955): Personal Influence. The part played by people in the flow of mass communications. Transaction Publishers.

- (2011) Personal Influence. The part played by people in the flow of mass communications. Transaction Publishers.

KING, C.W. (1965): "Fashion adoption: A rebuttal to the'trickle down'theory", en MCNEAL, James Dimensions of consumer behavior. Chicago: American Marketing Association, 1965, pp. 108-125.

KING, C.W. AND SUMMERS, J.O. (1970): “Overlap of opinion leadership across consumer product categories". Journal of Marketing Research. JSTOR, 7(1), pp. 43-50.

KING, C.W., TIGERT, D. J. AND RING, L. J. (1975): “Contemporary Fashion Theory and Retail Shopping Behavior: A Segmentation Analysis". in Marketing Educators' Conference of the American Marketing Association, Rochester, New York.

LAZARSFELD, P.F., BERELSON, B. AND GAUDET, H. (1944): The people's choice: how the voter makes up his mind in a presidential campaign. New York: Columbia University Press, 1968;

LIPOVESTKY, G. (1990): El imperio de lo efímero. La moda y su destino en las sociedades modernas. Barcelona: Anagrama.

MEYERSOHN, R. AND KATZ, E. (1957): "Notes on a natural history of fads". American Journal of Sociology. JSTOR, 62(6), pp. 594-601. doi: 10.1086/222108.

MATTHEWS, R. (2015): Contemporary Fashion Tastemakers: Starting Conversations that Matter. Catwalk: The Journal of Fashion, Beauty, and Style, 4(1), 51-70.

NAVARRO, G. M., \& GARCILLÁN LÓPEZ-RÚA, M. (2016): “La influencia de los blogs de moda en 
el comportamiento del consumidor. Un enfoque exploratorio". Vivat Academia, (135), 85-109.

PAÇO, A., \& OLIVEIRA, S. (2017): "Influence Marketing in the Fashion and Beauty Industry". Estudos em Comunicação, 1 (25).

PEDRONI, M., SÁDABA, T., \& SANMIGUEL, P. (2017): "Is the Golden Era of Fashion Blogs Over? An Analysis of the Italian and Spanish". Fields of Fashion Blogging. Fashion Tales Feeding the Imaginary, 105-124.

ROGERs, E.M. (1962): Difussion of innovations. New York: Free Press.

ROGERS, E.M. AND CARTANO, D.G. (1962): "Living Research Methods of Measuring Opinion Leadership," Public opinion quarterly. AAPOR, 26(3), pp. 435-441.

SÁDABA, T., \& SANMIGUEL, P. (2014): "Revisión de los Influentials en moda con la aparición de Internet: estudio del caso español a través de Stylelovely.com". Revista de Comunicación, 13,61 .

SAMMIS, K., NG, J., POMPONI, S., \& LINCOLN, C. (2015): Influencer Marketing For Dummies. Hoboken, NJ: For Dummies.

SANMIGUEL, P., \& SÁDABA, T. (2017): "Nice to be a fashion blogger, hard to be influential: An analysis based on personal characteristics, knowledge criteria, and social factors". Journal of Global Fashion Marketing, 1-19.

SCHAEFER, M. W. (2012): Return on influence : the revolutionary power of Klout, social scoring, and influence marketing. New York : McGraw-Hill, cop. 2012.

SIMMEL, G. (1923): "Filosofía de la moda". Revista de occidente. Pertenece a ARCE, (1), pp. 42-66.

SIMMEL, G. (1957): "Fashion". American Journal of Sociology. The University of Chicago Press, 62(6), pp. 541-558.

SOLIS, B., \& WEBBER, A. (2012): The Rise of Digital Influence. Altimeter Group. Recuperado a partir de www.altimetergroup.com

SPROLES, G. (1981): "Analyzing Fashion Life Cycles: Principles and Perspectives". Journal of Marketing, 45(4), pp. 116-124. doi: 10.2307/1251479.

SPROLES, G. AND KING, C. (1973): "The consumer fashion change agent: A theoretical conceptualization and empirical identification," en Institute for Research in the Behavioral, Economic, and Management Sciences, Purdue University. Paper No. 433, December

SUMMERS, J.O. (1970): "The identity of women's clothing fashion opinion leaders". Journal of Marketing Research. JSTOR, 7(2), pp. 178-185. 
TIGERT, D.J., KING, C. W. AND RING, L. (1980): “Fashion involvement: A cross-cultural comparative analysis". Advances in Consumer Research, 7(1), pp. 17-21.

TIGERT, D. J., RING, L. J. AND KING, C. W. (1976): "Fashion involvement and buying behavior: A methodological study". NA-Advances in Consumer Research Volume 03.

WIEDMANN, K.-P., HENNINGS, N., \& LANGNER, S. (2010): "Spreading the word of fashion: Identifying social influencers in fashion marketing". Journal of Global Fashion Marketing: Bridging Fashion and Marketing, 1(3), 142-153. http://doi.org/10.1080/20932685.2010.10593066

WORKMAN, J.E. AND JOHNSON, K.P. (1993): "Fashion opinion leadership, fashion innovativeness, and need for variety". Clothing and Textiles Research Journal. Sage Publications, 11(3), pp. 60-64. 